Case Report

\title{
Atypical Presentation of Sjögren-Larsson Syndrome
}

\author{
D. Papathemeli, ${ }^{1}$ A. Mataftsi, ${ }^{2}$ A. Patsatsi, ${ }^{1}$ D. Sotiriadis, ${ }^{1}$ M. Samouilidou, ${ }^{2}$ \\ S. Chondromatidou, ${ }^{3}$ and A. Evangeliou ${ }^{4}$ \\ ${ }^{1}$ 2nd Department of Dermatology and Venereology, Faculty of Medicine, Aristotle University of Thessaloniki, Papageorgiou General \\ Hospital, Thessaloniki, Greece \\ ${ }^{2}$ 2nd Department of Ophthalmology, Faculty of Medicine, Aristotle University of Thessaloniki, Papageorgiou General Hospital, \\ Thessaloniki, Greece \\ ${ }^{3}$ Department of Radiology, Papageorgiou General Hospital, Thessaloniki, Greece \\ ${ }^{4} 4$ th Department of Pediatrics, Faculty of Medicine, Aristotle University of Thessaloniki, Papageorgiou General Hospital, \\ Thessaloniki, Greece
}

Correspondence should be addressed to D. Papathemeli; dpapathem@live.com

Received 22 July 2017; Revised 14 September 2017; Accepted 27 September 2017; Published 18 October 2017

Academic Editor: Edvige Veneselli

Copyright $\odot 2017$ D. Papathemeli et al. This is an open access article distributed under the Creative Commons Attribution License, which permits unrestricted use, distribution, and reproduction in any medium, provided the original work is properly cited.

Sjögren-Larsson syndrome is a rare neurocutaneous disorder characterized by ichthyosis, spastic diplegia or tetraplegia, and intellectual disability. Herein, we describe a case of a Greek patient with ichthyosis and spasticity of the legs but with normal intelligence (IQ 95). This syndrome should be suspected when a child presents with ichthyosis and spastic diplegia or tetraplegia, even if intelligence is normal.

\section{Introduction}

Sjögren-Larsson syndrome (SLS) is a rare neurocutaneous disorder caused by deficient activity of fatty aldehyde dehydrogenase (FALDH) and is characterized by congenital ichthyosis, spastic diplegia or tetraplegia, and intellectual disability. This is a case of SLS with atypical presentation, expanding the phenotypic spectrum of the syndrome.

\section{Case Presentation}

A 3-year-old girl of Greek descent was referred to our hospital due to gait disturbance and undiagnosed skin lesions.

The patient presented bilateral spasticity of the legs. Acquisition of motor skills was delayed, as she achieved standing without support and walking at the age of 13 and 16 months, respectively. At the age of 16 months, she spoke $8-10$ words. There was no history of epileptic seizures. Upon examination, she presented with extensive hyperkeratosis and scaling of the skin especially on palms and soles and hyperpigmented skin flexures (Figures 1-3). Her hair and fingernails were normal. There was a history of recurrent chalazia. Her birth was at full-term (39 weeks), following an uneventful pregnancy. She is the fourth living child of a consanguineous couple who reported being third cousins, all other siblings having no significant medical history.

Height $(104 \mathrm{~cm})$, weight $(16.5 \mathrm{~kg})$, and head circumference $(53.5 \mathrm{~cm})$ were within normal age limits at presentation. Neurological evaluation revealed spastic diplegia of lower extremities, mild generalized increase in muscle tone, deep tendon reflexes of the lower extremities, and positive Babinski reflexes bilaterally. Intelligence was normal (IQ 95). Electroencephalopathy, otoacoustic emission test (OAE), abdominal and renal ultrasound, and X-ray of the chest and hips appeared to be normal.

Conventional magnetic resonance imaging (MRI) of the brain disclosed diffuse symmetrical high-intensity lesions in the deep white matter of the centrum semiovale and the frontal lobes and milder-intensity diffuse lesions in the posterior parietal lobes and the corpus callosum, while magnetic resonance spectroscopy (MRS) showed moderate increase of lipid and myoinositol levels. Other metabolites like $\mathrm{N}$-acetyl aspartate (NAA), choline (Cho), and creatine $(\mathrm{Cr})$ were within normal limits (Figures 4-6).

Fundoscopy was performed to search for signs of metabolic retinopathy, and no abnormal features were 


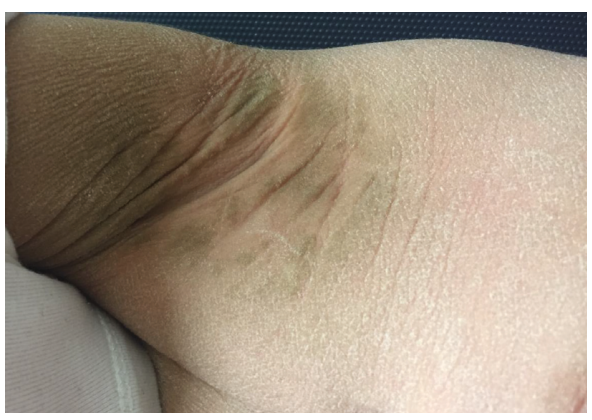

FIGURE 1: The patient presented with thickening and hyperpigmentation of both axillae.

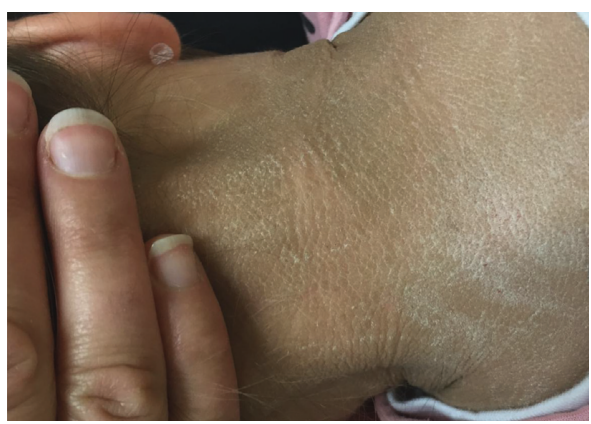

FIGURE 2: Brown scaly hyperpigmentation could also be seen on the nape of the neck.

detected in either eye. Absence of retinal deposits or other pathology was confirmed with optical coherence tomography of the macula. The patient had a history of recurrent chalazia. Cycloplegic retinoscopy revealed high refractive error (right eye $\mathrm{C}-4.50 \mathrm{D} \times 20^{\circ}$, left eye $\mathrm{C}-4.00 \mathrm{D} \times 170^{\circ}$ ). Visual acuity, measured with the ETDRS chart at 4 meters, was LogMAR 0.5 in the right eye and LogMAR 0.3 in the left eye with her glasses, which was presumed to be the result of refractive amblyopia and not of retinal pathology.

Histopathology of skin biopsy from the right axillary region and right thigh showed orthokeratotic hyperkeratosis, acanthosis, and papillomatosis of the stratum spinosum.

Clinical findings led to a differential diagnosis of SjögrenLarsson syndrome, Refsum disease, Rud syndrome, and Chanarin-Dorfman syndrome, and so genetic testing was performed. Sequencing of the polymerase chain reaction product using the exon-specific primers revealed a c.551C $>\mathrm{T}$ mutation of the ALDH3A2 gene on chromosome 17p11.2 in homozygous state and confirmed the diagnosis of SjögrenLarsson syndrome. Parents were tested and both were found to be heterozygous for this mutation. The patient was prescribed emollient baths and moisturizing creams, while physiotherapy was advised to improve spasticity.

No other clinical signs developed during three years of follow-up.

\section{Discussion}

Sjögren-Larsson syndrome is a rare autosomal recessive hereditary neurocutaneous disorder with a worldwide

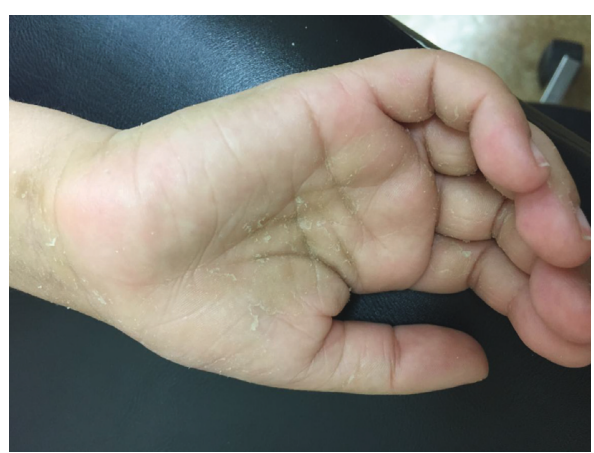

FIgURe 3: Palmoplantar keratoderma was also present.

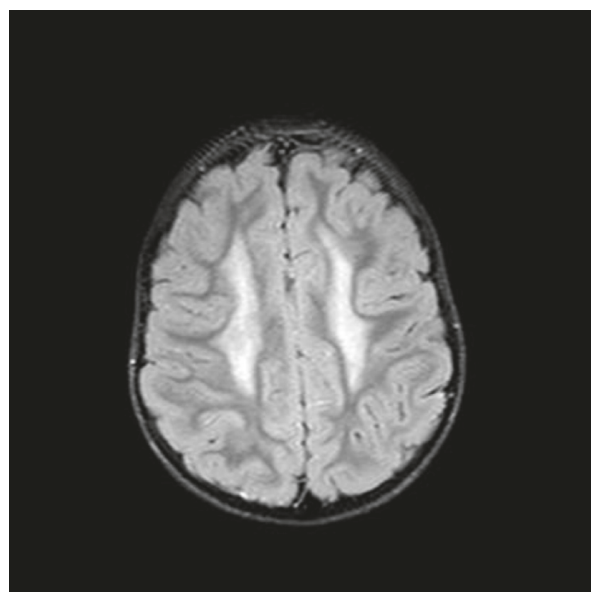

FIgURE 4: The deep white matter of the centrum semiovale bilateral demonstrates high signal intensity changes at Flair axial MR imaging.

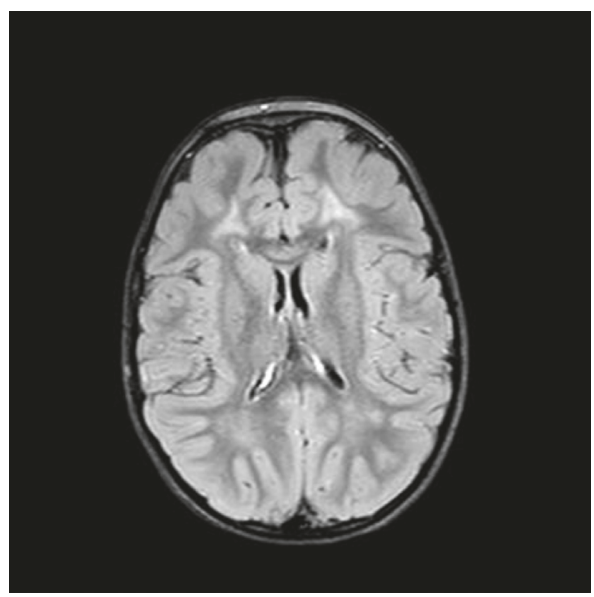

FIGURE 5: Flair axial MR image of the brain showing high-intensity lesions in the deep white matter of the frontal lobes and milderintensity lesions in the deep white matter of the posterior parietal lobes and the corpus callosum.

incidence of 0.4 per 100,000 people and was first described by Sjögren and Larsson in 1957 [1]. It is caused by deficient activity of fatty aldehyde dehydrogenase (FALDH), a component of the fatty alcohol nicotinamide adenine 


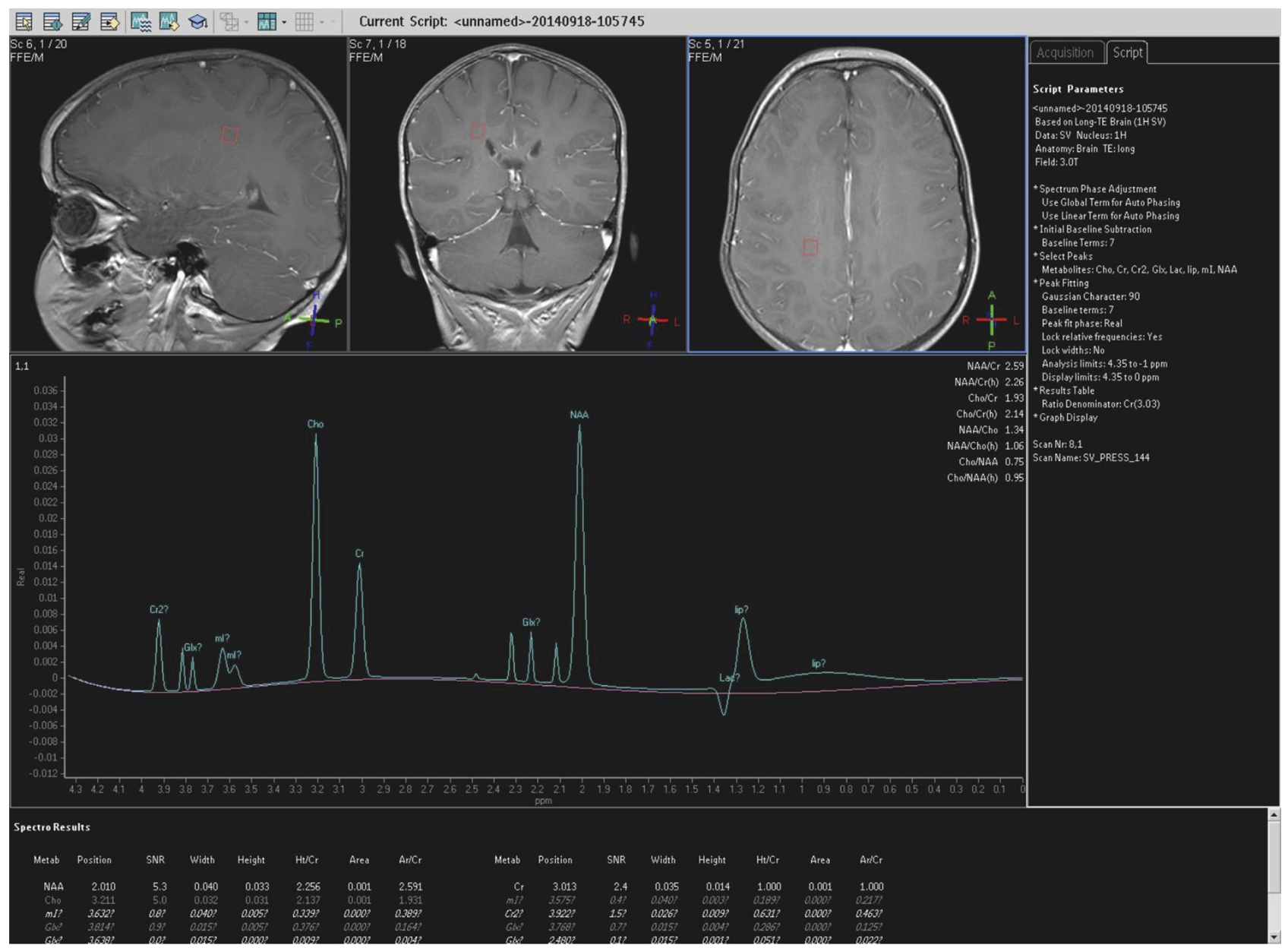

Figure 6: Magnetic resonance spectroscopy (MRS) showed moderate increase of lipid and myoinositol levels. Other metabolites like $\mathrm{N}$-acetyl aspartate (NAA), choline (Cho), and creatine (Cr) were within normal limits.

dinucleotide (NAD) oxidoreductase enzyme complex (FAO). FALDH deficiency results in the accumulation of fatty aldehydes and fatty alcohols in body tissues, which causes the symptoms [2]. This syndrome is characterized by the triad of congenital ichthyosis, spastic diplegia or tetraplegia, and mild to moderate intellectual disability (most patients show an intellectual coefficient of less than 50). Less common features are preterm birth, reduced visual acuity and photophobia, short stature, kyphoskoliosis, seizures, and delayed speech (spastic dysarthria may also be present) [3, 4].

FALDH deficiency affects epidermal functioning, and leaky water barrier causes ichthyosis. Cutaneous features are usually congenital or apparent during the neonatal period and become more pronounced overtime, as in our case $[2,3]$.

Neurological symptoms emerge by early childhood (first or second year of life). Later in adolescence, the neurological clinical picture stabilizes $[4,5]$. In our case, it is remarkable that the patient had normal intelligence (IQ 95).

Crystalline juvenile macular dystrophy, cystoid foveal atrophy, and lack of macular pigment are ocular features regarded as pathognomonic of Sjögren-Larsson syndrome but were not detected in our patient [6]. On the other hand, high refractive error and recurrent chalazia associated with meibomian gland dysfunction are signs that have not as yet been associated with the syndrome.

Diagnosis of SLS should be considered in children with simultaneous presence of congenital ichthyosis and debilitating neurological symptoms. However, the phenotype of SLS with the classical neurocutaneous features follows a typical age-dependent pattern and the full-blown phenotype becomes apparent after 2-3 years; therefore, SLS is usually not suspected until that age [3-5]. In our case, diagnosis was made at the age of 3 years.

Diagnosis of Sjögren-Larsson syndrome is confirmed by measurement of FALDH or fatty alcohol: NAD oxidoreductase in cultured skin fibroblasts [7] and/or sequence analysis of $A L D H 3 A 2$ gene on the locus 17p11.2. ALDH3A2 gene mutation tests are highly sensitive, do not require skin biopsy, and can complement or even replace FALDH enzymatic assays in SLS [8].

FALDH gene (also known as $A L D H 3 A 2$ ) which encodes fatty aldehyde dehydrogenase is located on chromosome $17 p 11.2$ and consists of 11 exons. More than 90 different mutations (amino acid substitutions, deletions, insertions, and splicing errors) have been found in patients with SLS. Missense mutations account for $38 \%$ of the known mutations in $A L D H 3 A 2$ and are scattered throughout the gene. 
Two mutations, c.1297-1298delGA and c.943C $>$ T, are considered to be the most frequent in Europe [8]. In our patient, the missense mutation c.551C $>\mathrm{T}$ was detected, which is responsible for replacement of threonine 184 by methionine (p.T184M) at the amino acid level of the FALDH enzyme. This mutation was first described by Rizzo et al. in 1999 as pathogenic and has been detected in Europe and Middle East [9]. It has been reported that the severity of clinical phenotype does not closely correlate with specific mutations. Even among siblings who share the same genotype, phenotype can vary significantly, due to genetic and environmental modifiers [10].

As with most inherited metabolic diseases, there is no curative therapy, and treatment is predominantly symptomatic. In our patient, ichthyosis improved significantly with abundant hydration with emollient baths, moisturizing creams, and keratolytic agents. In our case, prescription of acitretin was avoided because of the risk of premature epiphyseal closure. Physiotherapy improved ambulation and prevented contracture development. Studies have shown that zileuton, a 5-lipoxygenase inhibitor, has a potent advantageous effect upon pruritus in SLS patients, since it inhibits leukotriene formation [5, 11]. Future therapeutic strategies may include bezafibrates, carotenoids, and gene therapy [4, 5]. However, further research in this field is required.

\section{Conflicts of Interest}

The authors declare that there are no conflicts of interest regarding the publication of this article.

\section{Acknowledgments}

The authors thank the patient's parents for providing them permission to publish this case report.

\section{References}

[1] T. Sjögren and T. Larsson, "Oligophrenia in combination with congenital ichthyosis and spastic disorders; a clinical and genetic study," Acta Psychiatrica et Neurologica Scandinavica Supplementum, vol. 113, pp. 1-112, 1957.

[2] W. B. Rizzo, "Fatty aldehyde and fatty alcohol metabolism: review and importance for epidermal structure and function," Biochimica et Biophysica Acta (BBA)-Molecular and Cell Biology of Lipids, vol. 1841, no. 3, pp. 377-389, 2014.

[3] S. Jagell and S. Lidén, "Ichthyosis in the Sjögren-Larsson syndrome," Clinical Genetics, vol. 21, no. 4, pp. 243-252, 1982.

[4] A. Gånemo, S. Jagell, and A. Vahlquist, "Sjögren-Larsson syndrome: a study of clinical symptoms and dermatological treatment in 34 Swedish patients," Acta Dermato Venereologica, vol. 89, no. 1, pp. 68-73, 2009.

[5] J. Fuijkschot, T. Theelen, M. M. B. Seyger et al., "SjögrenLarsson syndrome in clinical practice," Journal of Inherited Metabolic Disease, vol. 35, no. 6, pp. 955-962, 2012.

[6] R. L. P. van der Veen, J. Fuijkschot, M. A. A. P. Willemsen, J. R. M. Cruysberg, T. T. J. M. Berendschot, and T. Theelen, "Patients with Sjögren-Larsson syndrome lack macular pigment," Ophthalmology, vol. 117, no. 5, pp. 966-971, 2010.
[7] W. B. Rizzo and D. A. Craft, "Sjögren-Larsson syndrome. Deficient activity of the fatty aldehyde dehydrogenase component of fatty alcohol: NAD+ oxidoreductase in cultured fibroblasts," Journal of Clinical Investigation, vol. 88, no. 5, pp. 1643-1648, 1991.

[8] W. B. Rizzo and G. Carney, "Sjögren-Larsson syndrome: diversity of mutations and polymorphisms in the fatty aldehyde dehydrogenase gene (ALDH3A2)," Human Mutation, vol. 26, no. 1, pp. 1-10, 2005.

[9] W. B. Rizzo, G. Carney, and Z. Lin, "The molecular basis of Sjögren-Larsson syndrome: mutation analysis of the fatty aldehyde dehydrogenase gene," American Journal of Human Genetics, vol. 65, no. 6, pp. 1547-1560, 1999.

[10] M. A. A. P. Willemsen, L. IJlst, P. M. Steijlen et al., "Clinical, biochemical and molecular genetic characteristics of 19 patients with the Sjögren-Larsson syndrome," Brain, vol. 124, no. 7, pp. 1426-1437, 2001.

[11] J. Fuijkschot, M. M. B. Seyger, D. E. T. Bastiaans, R. A. Wevers, N. Roeleveld, and M. A. A. P. Willemsen, "Zileuton for pruritus in Sjögren-Larsson syndrome: a randomized doubleblind placebo-controlled crossover trial," Acta Dermato Venereologica, vol. 96, no. 2, pp. 255-256, 2016. 


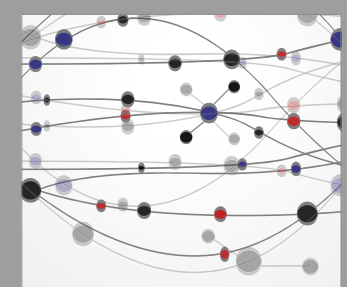

The Scientific World Journal
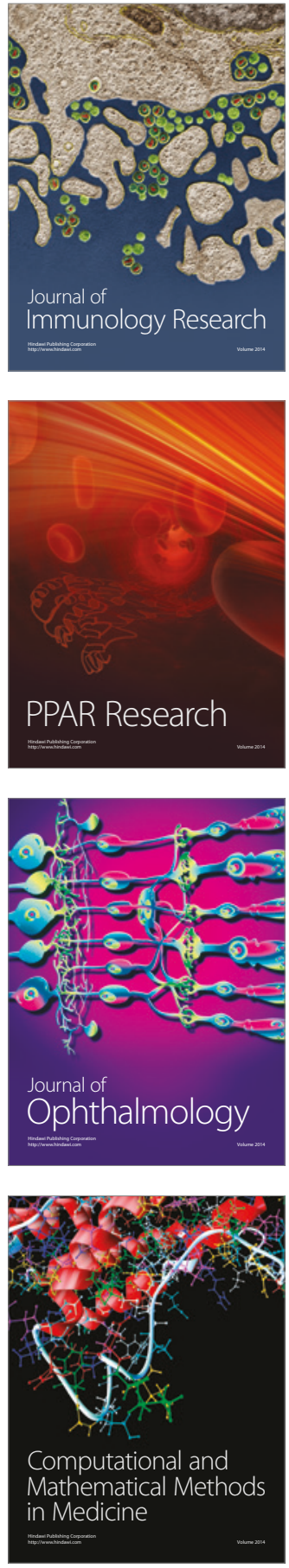

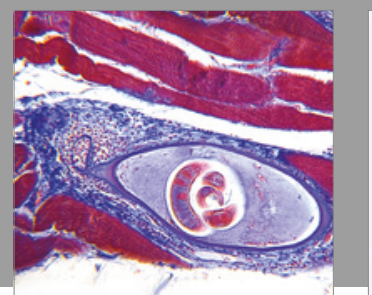

Gastroenterology Research and Practice
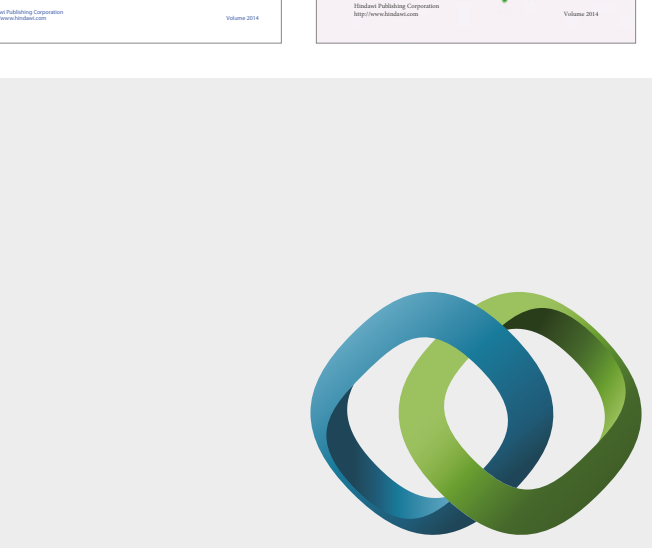

\section{Hindawi}

Submit your manuscripts at

https://www.hindawi.com
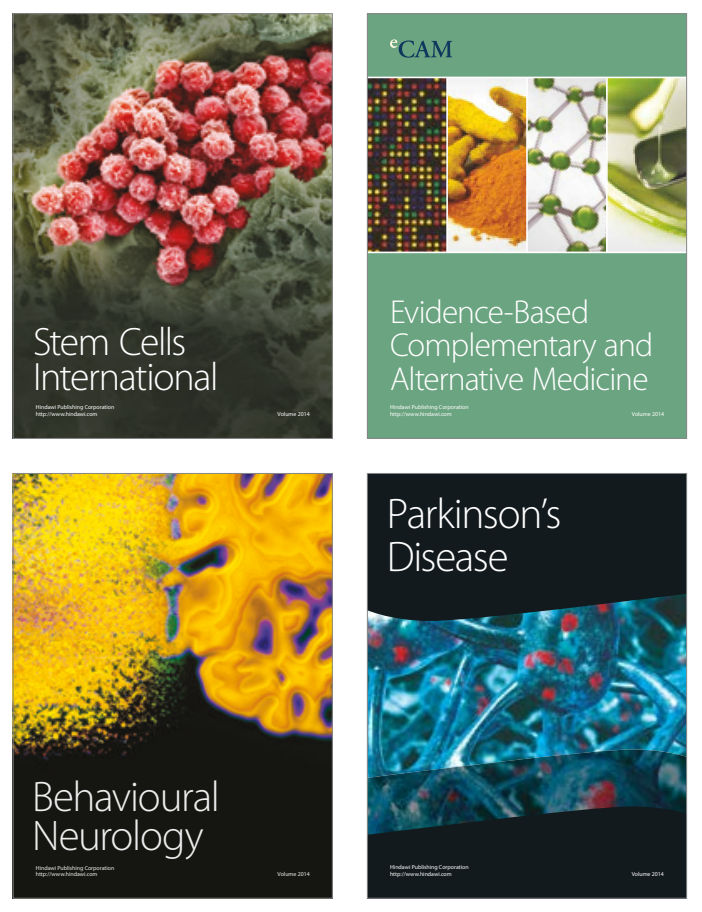
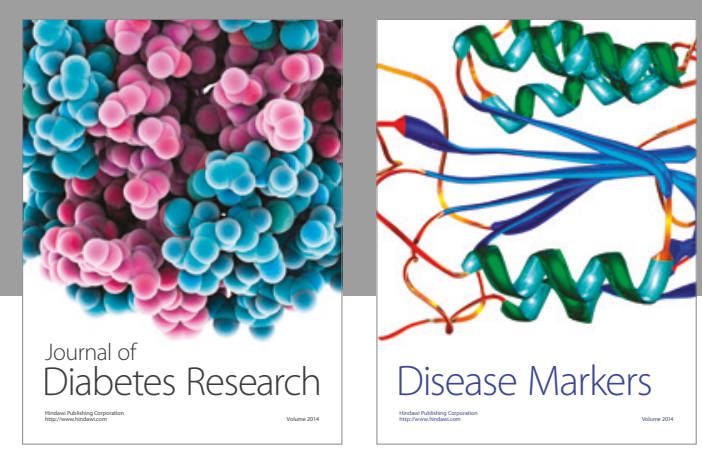

Disease Markers
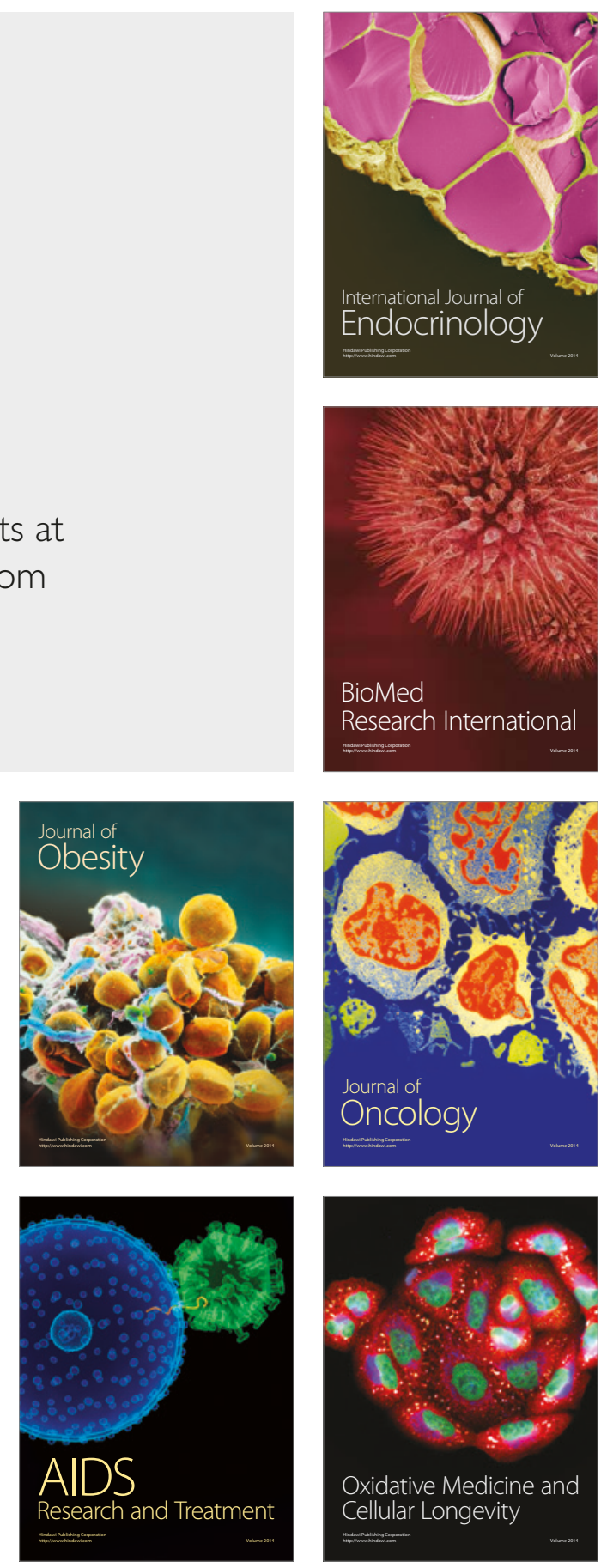